\title{
TLCD Parametric Optimization for the Vibration Control of Building Structures Based on Linear Matrix Inequality
}

\author{
Linsheng Huo, Chunxu Qu, and Hongnan Li \\ Faculty of Infrastructure Engineering, Dalian University of Technology, Dalian 116024, China \\ Correspondence should be addressed to Linsheng Huo; lshuo@dlut.edu.cn
}

Received 12 December 2013; Revised 26 May 2014; Accepted 29 May 2014; Published 18 June 2014

Academic Editor: Aderemi Oluyinka Adewumi

Copyright ( 2014 Linsheng Huo et al. This is an open access article distributed under the Creative Commons Attribution License, which permits unrestricted use, distribution, and reproduction in any medium, provided the original work is properly cited.

\begin{abstract}
Passive liquid dampers have been used to effectively reduce the dynamic response of civil infrastructures subjected to earthquakes or strong winds. The design of liquid dampers for structural vibration control involves the determination of the optimal parameters. This paper presents an optimal design methodology for tuned liquid column dampers (TLCDs) based on the $H_{\infty}$ control theory. A practical structure, Dalian Xinghai Financial Business Building, is used to illustrate the feasibility of the optimal procedure. The model of structure is built by the finite element method and simplified to the lumped mass model. To facilitate the design of TLCDs, the TLCD parametric optimization problem is transferred to the feedback controller design problem. Through the bounded real lemma, an optimization problem with bilinear matrix inequality (BMI) constraints is constructed to design a static output feedback $H_{\infty}$ controller. Iterative linear matrix inequality method is employed and it added some value range constraints to solve the BMI problem. After the TLCD parameters are optimized, the responses of displacement and acceleration in frequency domain and time domain are compared for the structure with and without TLCD. It is validated that the TLCD with the optimized parameters can make the structure satisfy the need for safety and comfort.
\end{abstract}

\section{Introduction}

Civil structural buildings can be damaged under heavy excitation, such as earthquake. The obvious vibration induced by some slight excitations, such as slight earthquakes and strong winds, can make the people in the building feel uncomfortable. Passive control is an effective strategy to suppress the vibration [1-4]. However, a lot of passive control devices are needed to be instrumented additionally. If there is already some device as a part of the building, it will not cost so much to deal with the vibration control. Tuned liquid column damper (TLCD) is first proposed by Sakai et al. [5], which can change its dynamics by tuning the mass, stiffness, and damping. So TLCD can be designed to make its dynamics be suitable for the vibration control of a building. In the tall building, the liquid tank filled with fresh water has to be instrumented for the fire protection or daily supply. TLCD can be set in the building as a liquid tank and also used for vibration control.

Considering Building 1 in Dalian Xinghai Financial Business Building, this paper presents a building vibration control example with TLCD. The finite element model is built and compared with the lumped mass model. It is found that these two models are pretty close. The TLCD dynamics is introduced, and the building dynamics with TLCD is discussed. Motivated by the method proposed by Moreno and Thomson [6], the formulation is derived to transfer the parametric optimization problem of TLCD to design a feedback controller problem.

For the feedback controller problem, this paper focuses on how to design a static output feedback $H_{\infty}$ controller. According to the bounded real lemma [7], designing the static $H_{\infty}$ controller is an optimization problem with bilinear matrix inequality (BMI) constraints. However, BMI is not a convex problem and cannot be solved with the general offthe-shelf packages as linear matrix inequality (LMI). To solve the static output feedback $H_{\infty}$ controller, Rubió-Massegú et al. [8] produced a simple expression of the controller, in which the corresponding symmetric variable matrix in the bounded real lemma is congruent with a block diagonal matrix. However, this variable matrix cannot represent all its possible results because there may exist some matrix 


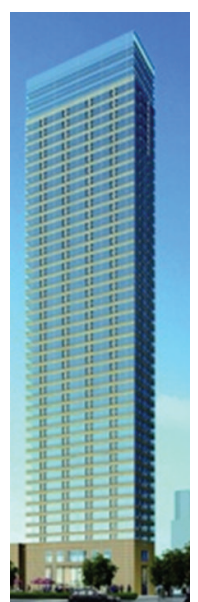

Figure 1: The buildings in Dalian Xinghai Bay Financial Business District.

that is not congruent with the block diagonal matrix. So the controller is conservative, and the desired controller may not be obtained. Zečević and Šiljak [9] present another method to design the static output feedback controller by making some transformations between the output matrix and controller matrix. Unfortunately, this method also supposed some relationship between output matrix and controller. So, the controller is also conservative. This paper employs the iterative linear matrix inequality (ILMI) method proposed by Cao et al. [10] and adds some value range restrictions into ILMI method. ILMI is solving a generalized eigenvalue minimization problem and does the iteration till satisfying the predetermined tolerance.

After the desired controller is obtained, the optimal parameters of TLCD are determined. The responses of the displacement and acceleration in frequency domain and time domain are compared to illustrate the performance of the optimized TLCD.

\section{Practical Model}

This paper considers the vibration control of a building subjected to the earthquake excitation, which has 46 stories and is 149.95 meters high, as shown in Figure 1.

The building has a frame-shear-wall structure and can be modeled by the finite element software ETABS. The finite element model contains 5472 joints, 13691 frame elements, and 4472 cell elements as shown in Figure 2.

Figure 2 displays the structural three-dimensional diagram as Figure 2(a) and structural top floor plan diagram as Figure 2(b). The first two vibration periods of the finite element model are $3.346829 \mathrm{~s}$ and $2.69537 \mathrm{~s}$. The corresponding vibration modes are shown in Figures 3(a) and 3(b).

However, the computation for the responses of the finite element model will cost a lot of time. Therefore, the lumped mass can be used to simplify the finite element model, which has 46 degrees of freedom (DOF) as shown in Figure 3(c). The structural masses are concentrated on each floor, and the interstory damping and stiffness between two lumped masses can be calculated from the finite element model.
TABLE 1: Comparison with difference of the period between two models.

\begin{tabular}{lcc}
\hline & $\begin{array}{c}\text { First vibration } \\
\text { period }\end{array}$ & $\begin{array}{c}\text { Second vibration } \\
\text { period }\end{array}$ \\
\hline Finite element model & 3.3468 & 2.6954 \\
Lumped mass model & 3.1312 & 2.6123 \\
Difference & $6.44 \%$ & $3.08 \%$ \\
\hline
\end{tabular}

To describe the difference between the finite element model and the lumped mass model, the vibration periods have been compared in Table 1.

The difference in the 3 rd row of Table 1 shows how different the two types of models are and can be calculated as the following expression:

$$
\frac{\left|T_{f}-T_{l}\right|}{T_{f}} \times 100 \%,
$$

where $T_{f}$ and $T_{l}$ are the vibration periods of the finite element model and lumped mass model, respectively. The above comparison illustrates that there is little difference between the finite element model and lumped mass model. So, the lumped mass model can be used to approximate the practical structure building. In Table 1, the first and second vibration periods are the first mode along $Y$ direction and the first mode along $X$ direction, respectively. It means that the structural stiffness along $X$ direction is larger than the one along $Y$ direction; that is to say, the structure along $Y$ direction is weaker than that along $X$ direction. So, the lumped mass model can be built only considering the stiffness and damping along $Y$ direction. The structure is subjected to an earthquake, whose dynamics can be formulated as follows:

$$
\mathbf{M} \ddot{\mathbf{x}}+\mathbf{C} \dot{\mathbf{x}}+\mathbf{K x}=-\mathbf{M} \ddot{x}_{g},
$$

where

$$
\begin{gathered}
\mathbf{M}=\left[\begin{array}{ccc}
m_{1} & 0 & 0 \\
0 & \ddots & 0 \\
0 & 0 & m_{n}
\end{array}\right], \\
\mathbf{C}=\left[\begin{array}{ccc}
c_{1}+c_{2} & -c_{2} & 0 \\
-c_{2} & \ddots & -c_{n} \\
0 & -c_{n} & c_{n}
\end{array}\right], \\
\mathbf{K}=\left[\begin{array}{ccc}
k_{1}+k_{2} & -k_{2} & 0 \\
-k_{2} & \ddots & -k_{n} \\
0 & -k_{n} & k_{n}
\end{array}\right],
\end{gathered}
$$

where the elements $m_{i}, c_{i}$, and $k_{i}$ in matrices $\mathbf{M}, \mathbf{C}$, and $\mathbf{K}$ are the mass, damping, and stiffness of the $i$ th floor $(i=$ $1, \ldots, n, n=46)$. It should be noted that matrices $\mathbf{C}$ and $\mathrm{K}$ are calculated along $Y$ direction. $\ddot{x}_{g}$ is the unidirectional earthquake acceleration. The relative displacement vector $\mathbf{x} \epsilon$ $\mathfrak{R}^{n \times 1}$ is defined as

$$
\mathbf{x}=\left[\begin{array}{llll}
x_{1} & x_{2} & \cdots & x_{n}
\end{array}\right]^{\mathrm{T}}
$$

For the tall building, the structural design proposed does not only make the building satisfy safety requirement during 


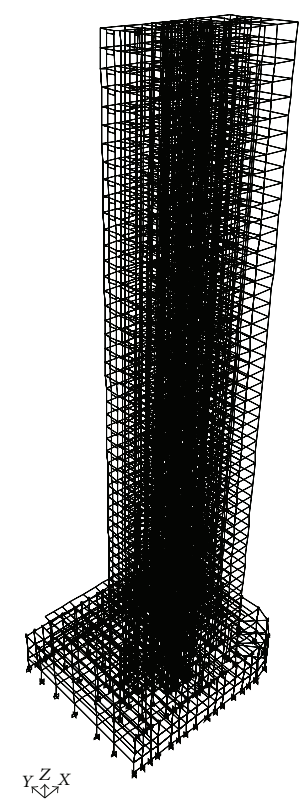

(a)

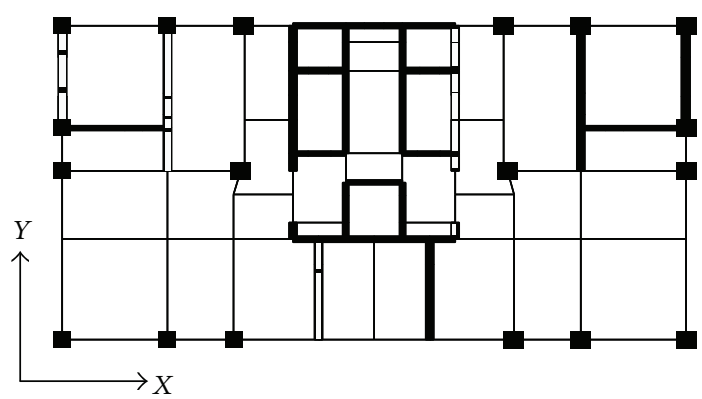

(b)

FIGURE 2: Structural diagram: (a) structural three-dimensional diagram; (b) structural top floor plan diagram.

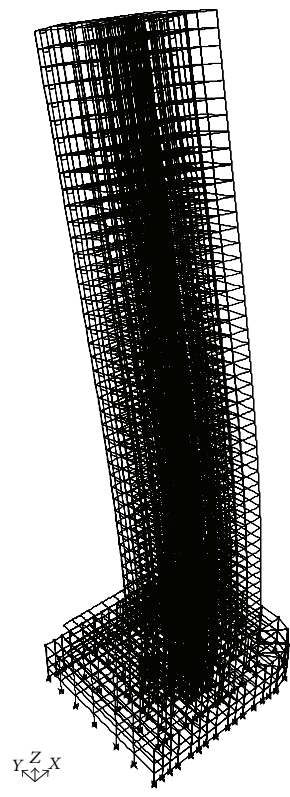

(a)

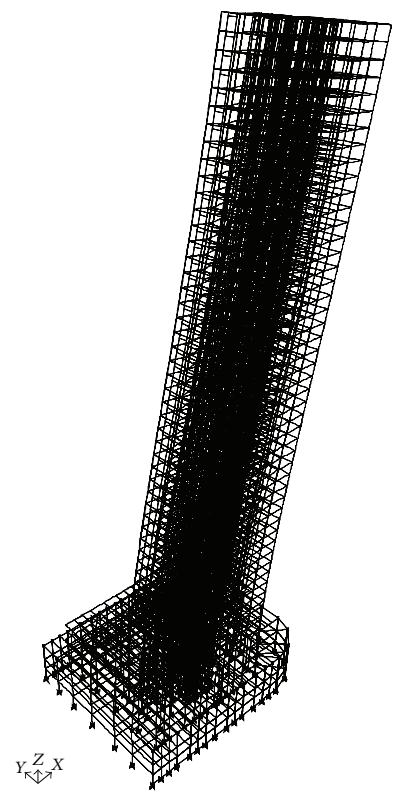

(b)

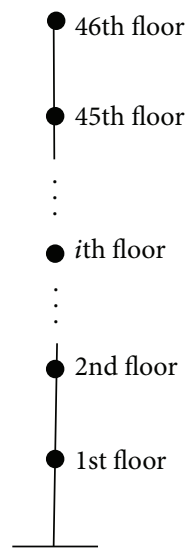

(c)

FIgURE 3: Structural diagram: (a) the first vibration mode; (b) the second vibration mode; (c) the lumped mass model.

the earthquake excitation but also make the people in the building feel comfortable during the slight external excitation, such as wind excitation. The safe requirements mean that the interstory drifts of the vibrating building should be smaller. The comfortable requirement needs the acceleration of the building to be smaller.

A liquid tank with fresh water has to be set in a tall building, which would be used for the fire protection or daily supply. So, the structural vibration can be suppressed by the liquid tank with appropriate dynamics. The dynamics of the liquid tank can be designed by the tank shapes. This paper employs the tank with the shape of two columns that is called the tuned liquid column damper (TLCD).

\section{TLCD Design}

3.1. The Dynamics of the Structure with TLCD. The structure of the tuned liquid column damper (TLCD) is shown as 


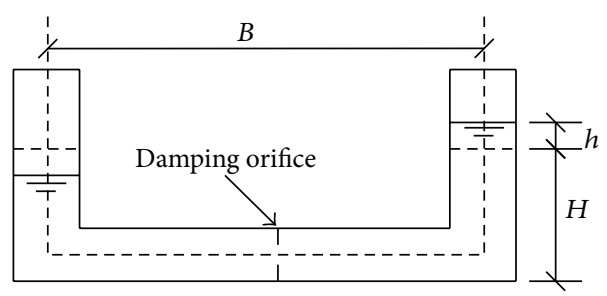

(a)

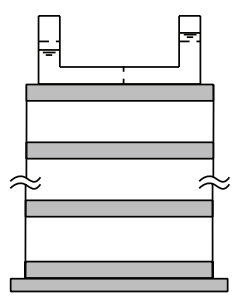

(b)

FIGURE 4: Tuned liquid column damper system: (a) tuned liquid column damper; (b) the building with tuned liquid column damper.

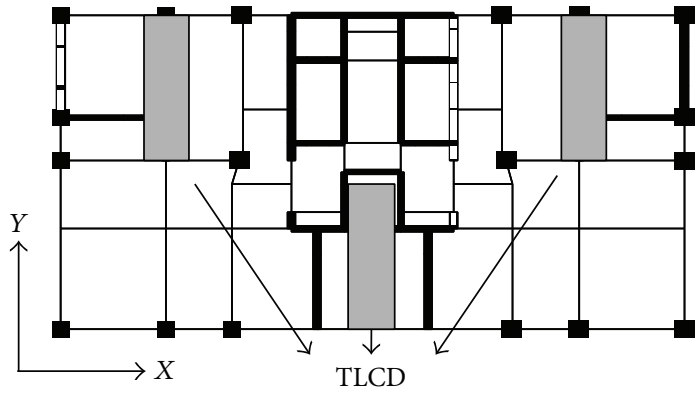

Figure 5: Deployment of three TLCDs on the top floor.

Figure 4(a). When it is located on the top floor of the building as shown in Figure 4(b), the TLCD dynamics can be described as the following equation, which is derived by Lagrange's method:

$$
\rho A(2 H+B) \ddot{h}+\frac{1}{2} \rho A \xi|\dot{h}| \dot{h}+2 \rho A g h=-\rho A B\left(\ddot{x}_{46}+\ddot{x}_{g}\right),
$$

where $\rho$ is the liquid density, $h$ is the displacement of the liquid in the damper, $H$ is the vertical length of the liquid column when the liquid is quiescent, $A$ is the cross-sectional area of the tube, $B$ is the horizontal length of the column, and $\xi$ is the head-loss coefficient. $\ddot{x}_{g}$ is the unidirectional earthquake acceleration. $\ddot{x}_{46}$ is the relative acceleration (with respect to the ground) for the top floor of the building. Equation (5) can be simplified as

$$
m_{T} \ddot{h}+c_{T} \dot{h}+k_{T} h=-\alpha m_{T}\left(\ddot{x}_{46}+\ddot{x}_{g}\right)
$$

where $m_{T}=\rho A L$ means the mass of liquid in the tube, $c_{T}=$ $2 m_{T} \omega_{T} \zeta_{T}$ is the equivalent damping of the liquid damper, $\omega_{T}$ is the first resonant frequency of TLCD, $\zeta_{T}$ is the damping ratio of the liquid damper, $k_{T}=2 \rho \mathrm{Ag}$ expresses the stiffness of the liquid column, $g$ is the gravitational constant, and $\alpha=$ $B / L$ denotes the length ratio. According to (4) and (6), the dynamics of the structure instrumented with TLCD on the top floor can be formulated as

$$
\mathbf{M}_{s} \ddot{\mathbf{q}}+\mathbf{C}_{s} \dot{\mathbf{q}}+\mathbf{K}_{s} \mathbf{q}=\mathbf{T}_{w} \ddot{x}_{g},
$$

where

$$
\begin{gathered}
\mathbf{M}_{s}=\left[\begin{array}{cccc}
m_{1} & 0 & 0 & 0 \\
0 & \ddots & 0 & 0 \\
0 & 0 & m_{n}+m_{T} & \alpha m_{T} \\
0 & 0 & \alpha m_{T} & m_{T}
\end{array}\right], \\
\mathbf{C}_{s}=\left[\begin{array}{cccc}
c_{1}+c_{2} & -c_{2} & 0 & 0 \\
-c_{2} & \ddots & -c_{n} & 0 \\
0 & -c_{n} & c_{n} & 0 \\
0 & 0 & 0 & c_{T}
\end{array}\right],
\end{gathered}
$$$$
\mathbf{K}_{s}=\left[\begin{array}{cccc}
k_{1}+k_{2} & -k_{2} & 0 & 0 \\
-k_{2} & \ddots & -k_{n} & 0 \\
0 & -k_{n} & k_{n} & 0 \\
0 & 0 & 0 & k_{T}
\end{array}\right] \text {, }
$$

$$
\mathbf{T}_{w}=-\left[\begin{array}{cccc}
m_{1} & 0 & 0 & 0 \\
0 & \ddots & 0 & 0 \\
0 & 0 & m_{n}+m_{T} & 0 \\
0 & 0 & 0 & \alpha m_{T}
\end{array}\right] \text {, }
$$

$$
\mathbf{q}=\left[\begin{array}{llll}
q_{1} & \cdots & q_{n} & q_{n+1}
\end{array}\right]^{\mathrm{T}}=\left[\begin{array}{llll}
x_{1} & \cdots & x_{n} & h
\end{array}\right]^{\mathrm{T}} \in \mathfrak{R}^{(n+1) \times 1} .
$$

From (7), it is found that the dynamics of the structure instrumented with TLCD can be influenced by the TLCD parameters, such as $m_{T}, c_{T}$, and $k_{T}$. When a TLCD is needed to be designed for a building structure, the value ranges for the mass ratio, frequency tuning ratio, and damping ratio are always given first, which can be determined uniquely by $m_{T}, c_{T}$, and $k_{T}$. In practical engineering, mass ratio is usually predetermined to satisfy the architectural requirements. Therefore, only frequency tuning ratio and damping ratio should be optimized during the TLCD designing. There are the following relationships with $m_{T}, c_{T}$, and $k_{T}$ :

$$
\begin{gathered}
\tau=\frac{\omega_{T}}{\omega_{s}}=\frac{\sqrt{k_{T}}}{\omega_{s} \sqrt{m_{T}}}, \\
\zeta_{T}=\frac{c_{T}}{2 m_{T} \omega_{T}}=\frac{c_{T}}{2 \sqrt{m_{T} k_{T}}},
\end{gathered}
$$

where $\tau$ is the frequency tuning ratio, $\omega_{s}$ is the first resonant frequency of the building, and $\zeta_{T}$ is the TLCD damping ratio as defined above. The TLCD designing objective is to optimize the parameters $\tau$ and $\zeta_{T}$ such that the structural vibration is reduced and satisfies the need for safety and comfort. From (9), $c_{T}$ and $k_{T}$ can replace the parameters $\tau$ and $\zeta_{T}$ to be optimized. 
3.2. Transfer Parametric Optimization to Controller Design. In order to optimize the parameters $c_{T}$ and $k_{T}$ in (8), they are separated from the matrices $\mathbf{C}_{s}$ and $\mathbf{K}_{s}$. Equation (7) is equal to the following expression:

$$
\mathbf{M}_{s} \ddot{\mathbf{q}}+\overline{\mathbf{C}} \dot{\mathbf{q}}+\overline{\mathbf{K}} \mathbf{q}=\mathbf{T}_{w} \ddot{x}_{g}+\mathbf{T}_{u} p(t),
$$

where

$$
\begin{gathered}
\overline{\mathbf{C}}=\left[\begin{array}{cccc}
c_{1}+c_{2} & -c_{2} & 0 & 0 \\
-c_{2} & \ddots & -c_{n} & 0 \\
0 & -c_{n} & c_{n} & 0 \\
0 & 0 & 0 & 0
\end{array}\right], \\
\overline{\mathbf{K}}=\left[\begin{array}{cccc}
k_{1}+k_{2} & -k_{2} & 0 & 0 \\
-k_{2} & \ddots & -k_{n} & 0 \\
0 & -k_{n} & k_{n} & 0 \\
0 & 0 & 0 & 0
\end{array}\right], \\
\mathbf{T}_{u}=\left[\begin{array}{lll}
\mathbf{0}_{1 \times n} & -1
\end{array}\right]^{\mathrm{T}}, \\
p(t)=\left[\begin{array}{ll}
k_{T} & c_{T}
\end{array}\right]\left[\begin{array}{l}
h \\
\dot{h}
\end{array}\right] .
\end{gathered}
$$

Equation (10) can be recognized as the structure with the mass matrix $\mathbf{M}_{s}$, damping matrix $\overline{\mathbf{C}}$, and stiffness matrix $\overline{\mathbf{K}}$ subjected to the earthquake excitation $\ddot{x}_{g}$ and the control force $p(t)$. An appropriate control force $p(t)$ can suppress the structural vibration. From (12), the value of the control force can be decided by the matrix $\left[\begin{array}{ll}k_{T} & c_{T}\end{array}\right]$ and would vary with the TLCD state $\left[\begin{array}{ll}h & \dot{h}\end{array}\right]^{\mathrm{T}}$. Equation (10) can be represented by the augmented system in state-space as

$$
\begin{gathered}
\dot{\mathbf{z}}=\mathbf{A} \mathbf{z}+\mathbf{B}_{1} \ddot{x}_{g}+\mathbf{B}_{2} p(t), \\
\mathbf{y}_{z}=\mathbf{C}_{1} \mathbf{z}+\mathbf{D}_{11} \ddot{x}_{g}+\mathbf{D}_{12} p(t), \\
\mathbf{y}_{m}=\mathbf{C}_{2} \mathbf{z}+\mathbf{D}_{21} \ddot{x}_{g}+\mathbf{D}_{22} p(t),
\end{gathered}
$$

where

$$
\mathbf{z}=\left[\begin{array}{ll}
\mathbf{q} & \dot{\mathbf{q}}
\end{array}\right]^{\mathrm{T}} ; \quad \mathbf{y}_{m}=\left[\begin{array}{ll}
h & \dot{h}
\end{array}\right]^{\mathrm{T}}
$$

Matrices $\mathbf{C}_{1}, \mathbf{D}_{11}$, and $\mathbf{D}_{12}$ can be determined according to objective output $\mathbf{y}_{z}$, which is always the combination of the structural interstory drifts and the weighted control force. Other matrices in (13) are denoted as

$$
\begin{aligned}
& {\left[\begin{array}{c|c|c}
\mathbf{A} & \mathbf{B}_{1} & \mathbf{B}_{2} \\
\hline \mathbf{C}_{2} & \mathbf{D}_{21} & \mathbf{D}_{22}
\end{array}\right]}
\end{aligned}
$$

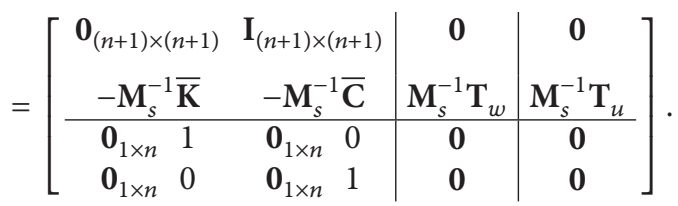

The control force $p(t)$ in (12) can be rewritten as

$$
p(t)=\mathbf{G y}_{m}=\left[\begin{array}{ll}
k_{T} & c_{T}
\end{array}\right]\left[\begin{array}{l}
h \\
\dot{h}
\end{array}\right] .
$$

So, the TLCD parametric optimization problem is transferred to the controller design problem, that is, to design a controller G to suppress the structural vibration efficiently.

3.3. $H_{\infty}$ Static Output Feedback Controller Design. Taking (16) into (13) to complete the feedback loop, the closed-loop system can be represented as

$$
\begin{gathered}
\dot{\mathbf{z}}=\mathbf{A}_{\mathrm{CL}} \mathbf{z}+\mathbf{B}_{\mathrm{CL}} \ddot{x}_{g}, \\
\mathbf{y}_{z}=\mathbf{C}_{\mathrm{CL}} \mathbf{z}+\mathbf{D}_{\mathrm{CL}} \ddot{x}_{g},
\end{gathered}
$$

where

$$
\begin{gathered}
\mathbf{A}_{\mathrm{CL}}=\mathbf{A}+\mathbf{B}_{2} \mathbf{G C}_{2} \\
\mathbf{B}_{\mathrm{CL}}=\mathbf{B}_{1}+\mathbf{B}_{2} \mathbf{G D}_{21} \\
\mathbf{C}_{\mathrm{CL}}=\mathbf{C}_{1}+\mathbf{D}_{12} \mathbf{G C}_{2} \\
\mathbf{D}_{\mathrm{CL}}=\mathbf{D}_{11}+\mathbf{D}_{12} \mathbf{G D}_{21} .
\end{gathered}
$$

This paper focuses on how to design a static output feedback $H_{\infty}$ controller $\mathbf{G}$ in (16). Some concepts about $H_{\infty}$ can refer to [11]. According to the bounded real lemma [7], an $H_{\infty}$ controller can be designed to stabilize the closed-loop system in (17) and make the transfer function norm of the closedloop system smaller than a given scalar $\gamma$, if and only if there exists a symmetric positive definite matrix $\mathbf{X}_{\mathrm{CL}}>0$ to satisfy the following matrix inequality:

$$
\left[\begin{array}{ccc}
\mathbf{A}_{\mathrm{CL}}^{\mathrm{T}} \mathbf{X}_{\mathrm{CL}}+\mathbf{X}_{\mathrm{CL}} \mathbf{A}_{\mathrm{CL}} & \mathbf{X}_{\mathrm{CL}} \mathbf{B}_{\mathrm{CL}} & \mathbf{C}_{\mathrm{CL}}^{\mathrm{T}} \\
\mathbf{B}_{\mathrm{CL}}^{\mathrm{T}} \mathbf{X}_{\mathrm{CL}} & -\gamma \mathbf{I} & \mathbf{D}_{\mathrm{CL}}^{\mathrm{T}} \\
\mathbf{C}_{\mathrm{CL}} & \mathbf{D}_{\mathrm{CL}} & -\gamma \mathbf{I}
\end{array}\right]<\mathbf{0}
$$

Substitute (18) into (19):

$$
\left[\begin{array}{ccc}
\left(\mathbf{A}+\mathbf{B}_{2} \mathbf{G C}_{2}\right)^{\mathrm{T}} \mathbf{X}_{\mathrm{CL}}+\mathbf{X}_{\mathrm{CL}}\left(\mathbf{A}+\mathbf{B}_{2} \mathbf{G C}_{2}\right) & \mathbf{X}_{\mathrm{CL}}\left(\mathbf{B}_{1}+\mathbf{B}_{2} \mathbf{G D}_{21}\right) & \left(\mathbf{C}_{1}+\mathbf{D}_{12} \mathbf{G C}_{2}\right)^{\mathrm{T}} \\
\left(\mathbf{B}_{1}+\mathbf{B}_{2} \mathbf{G D}_{21}\right)^{\mathrm{T}} \mathbf{X}_{\mathrm{CL}} & -\gamma \mathbf{I} & \left(\mathbf{D}_{11}+\mathbf{D}_{12} \mathbf{G D}_{21}\right)^{\mathrm{T}} \\
\mathbf{C}_{1}+\mathbf{D}_{12} \mathbf{G C} \mathbf{C}_{2} & \mathbf{D}_{11}+\mathbf{D}_{12} \mathbf{G D}_{21} & -\gamma \mathbf{I}
\end{array}\right]<\mathbf{0} .
$$


In practical engineering, there are always some value ranges for the frequency tuning ratio $\tau$ and damping ratio $\zeta_{T}$. So, the value ranges for $k_{T}$ and $c_{T}$ in $\mathbf{G}$ can be calculated from (9) and formulated as

$$
\begin{array}{cc}
c_{T}=\mathbf{L}_{c} \mathbf{G R}_{c}, & c_{T}^{l} \leq \mathbf{L}_{c} \mathbf{G} \mathbf{R}_{c} \leq c_{T}^{u}, \\
k_{T}=\mathbf{L}_{k} \mathbf{G R}_{k}, & k_{T}^{l} \leq \mathbf{L}_{k} \mathbf{G R}_{k} \leq k_{T}^{u},
\end{array}
$$

where

$$
\begin{array}{ll}
\mathbf{L}_{c}=[1], & \mathbf{R}_{c}=\left[\begin{array}{ll}
0 & 1
\end{array}\right]^{\mathrm{T}}, \\
\mathbf{L}_{k}=[1], & \mathbf{R}_{k}=\left[\begin{array}{ll}
1 & 0
\end{array}\right]^{\mathrm{T}} .
\end{array}
$$

In (21), $c_{T}^{u}$ and $c_{T}^{l}$ are denoted as the upper and lower boundary for $c_{T}$ and $k_{T}^{u}$ and $k_{T}^{l}$ are the upper and lower boundary for $k_{T}$, respectively. So, the controller $\mathbf{G}$ should be searched with the constraints (20) and (21). However, it is a bilinear matrix inequality problem, which is nonconvex. Rubió-Massegú et al. [8] and Zečević and Šiljak [9] proposed new approaches to deal with the static output feedback problem, respectively. But the controllers obtained by these two methods are both conservative due to the predefined restrictions. This paper employs the iterative linear matrix inequality (ILMI) method proposed by Cao et al. [10] and adds the constraints (21) into this method to numerically calculate the static output feedback controller G. In order to use ILMI method, (20) should be rewritten into another form:

$$
(\overline{\mathbf{A}}+\overline{\mathbf{B}} \mathbf{G} \overline{\mathbf{C}})^{\mathrm{T}} \mathbf{P}+\mathbf{P}(\overline{\mathbf{A}}+\overline{\mathbf{B}} \mathbf{G} \overline{\mathbf{C}})<\mathbf{0},
$$

where

$$
\begin{array}{cc}
\mathbf{P}=\left(\begin{array}{ccc}
\mathbf{X}_{\mathbf{C L}} & \mathbf{0} & \mathbf{0} \\
\mathbf{0} & \mathbf{I} & \mathbf{0} \\
\mathbf{0} & \mathbf{0} & \mathbf{I}
\end{array}\right), & \overline{\mathbf{A}}=\left(\begin{array}{ccc}
\mathbf{A} & \mathbf{B}_{1} & \mathbf{0} \\
\mathbf{0} & -\gamma \mathbf{I} / 2 & \mathbf{0} \\
\mathbf{C}_{1} & \mathbf{D}_{11} & -\gamma \mathbf{I} / 2
\end{array}\right), \\
\overline{\mathbf{B}}=\left(\begin{array}{c}
\mathbf{B}_{2} \\
\mathbf{0} \\
\mathbf{D}_{12}
\end{array}\right), & \overline{\mathbf{C}}=\left(\begin{array}{lll}
\mathbf{C}_{2} & \mathbf{D}_{21} & \mathbf{0}
\end{array}\right) .
\end{array}
$$

Equation (23) is a Lyapunov inequality, which means that there is a static output feedback to stabilize the system $(\overline{\mathbf{A}}, \overline{\mathbf{B}}, \overline{\mathbf{C}})$. So, the ILMI method can be used for the system $(\overline{\mathbf{A}}, \overline{\mathbf{B}}, \overline{\mathbf{C}})$ and summarized as follows.

Step 1. According to the value range of the parameters $\tau$ and $\zeta_{T}$, set the lower boundaries $c_{T}^{l}$ and $k_{T}^{l}$ and upper boundaries $c_{T}^{u}$ and $k_{T}^{u}$ for $c_{T}$ and $k_{T}$, respectively. Select $\mathbf{Q}>\mathbf{0}$ and solve $\mathbf{P}$ from the following algebraic Riccati equation:

$$
\overline{\mathbf{A}}^{\mathrm{T}} \mathbf{P}+\mathbf{P} \overline{\mathbf{A}}-\mathbf{P} \overline{\mathbf{B B}}^{\mathrm{T}} \mathbf{P}+\mathbf{Q}=\mathbf{0} .
$$

Set $i=1$ and $\mathbf{X}_{1}=\mathbf{P}$.

Step 2. Solve the following convex problem for $\mathbf{P}_{i}$, G, and $\alpha_{i}$ :

$$
\begin{aligned}
& \text { Minimize } \alpha_{i} \\
& \text { s.t. } \quad\left(\begin{array}{cc}
\overline{\mathbf{A}}^{\mathrm{T}} \mathbf{P}_{i}+\mathbf{P}_{i} \overline{\mathbf{A}}-\mathbf{X}_{i} \overline{\mathbf{B B}}^{\mathrm{T}} \mathbf{P}_{i}-\mathbf{P}_{i} \overline{\mathbf{B B}}^{\mathrm{T}} \mathbf{X}_{i}+\mathbf{X}_{i} \overline{\mathbf{B B}}^{\mathrm{T}} \mathbf{X}_{i}-\alpha_{i} \mathbf{P}_{i}\left(\overline{\mathbf{B}}^{\mathrm{T}} \mathbf{P}_{i}+\mathbf{G} \overline{\mathbf{C}}\right)^{\mathrm{T}} \\
\overline{\mathbf{B}}^{\mathrm{T}} \mathbf{P}_{i}+\mathbf{G} \overline{\mathbf{C}} & -\mathbf{I}
\end{array}\right)<\mathbf{0} \\
& \mathbf{P}_{i}=\mathbf{P}_{i}^{\mathrm{T}}>\mathbf{0} \\
& c_{T}^{l} \leq \mathbf{L}_{c} \mathbf{G R}_{c} \leq c_{T}^{u}, \quad k_{T}^{l} \leq \mathbf{L}_{k} \mathbf{G R}_{k} \leq k_{T}^{u} .
\end{aligned}
$$

Set $\alpha_{i}^{*}=$ the minimized $\alpha_{i}$.

Step 3. If $\alpha_{i}^{*} \leq 0, \mathrm{G}$ is the desired static output feedback gain in (16). Iteration can be stopped.

Step 4. Solve the variable matrices $\mathbf{P}_{i}$ and $\mathbf{G}$ by minimizing trace $\left(\mathbf{P}_{i}\right)$ with the constraints in (26), where $\alpha_{i}=\alpha_{i}^{*}$. Set $\mathbf{P}_{i}^{*}$ $=$ the solution $\mathbf{P}_{i}$.

Step 5. Compare $\mathbf{X}_{i}$ and $\mathbf{P}_{i}^{*}$. If $\left\|\mathbf{X}_{i}-\mathbf{P}_{i}^{*}\right\|$ is smaller than a given tolerance, go to Step 6; else set $i=i+1$ and $\mathbf{X}_{i}=\mathbf{P}_{i}^{*}$, and then go to Step 2.

Step 6. The system may not be stabilized through the output feedback gain in (20) with the constraints (21). Stop the iteration.
It should be noted that the matrix $\mathbf{Q}$ in (25) of Step 1 can influence the convergence of the ILMI method. If the iteration cannot be converged, another $\mathbf{Q}$ should be chosen. $\mathbf{Q}$ is always set to the identity matrix such that the iteration may obtain a good convergence. Another notice is that the convex problem in Step 2 is a generalized eigenvalue minimization problem. It can be solved through the PEMBMI solver in Yalmip [12].

\section{Calculation Results}

For the building modeled in the first section, the TLCDs can be instrumented on the top floor and used for fire protection or daily supply, whose deployment is shown in Figure 5.

For the three TLCDs, they are first assumed to be one TLCD for the purpose of simplicity during optimization. 


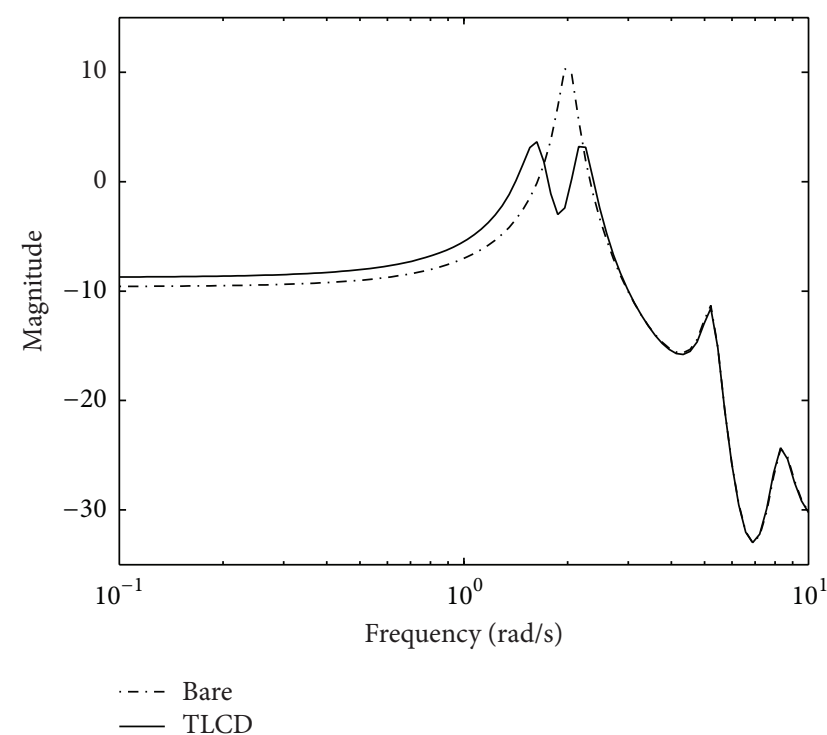

(a)

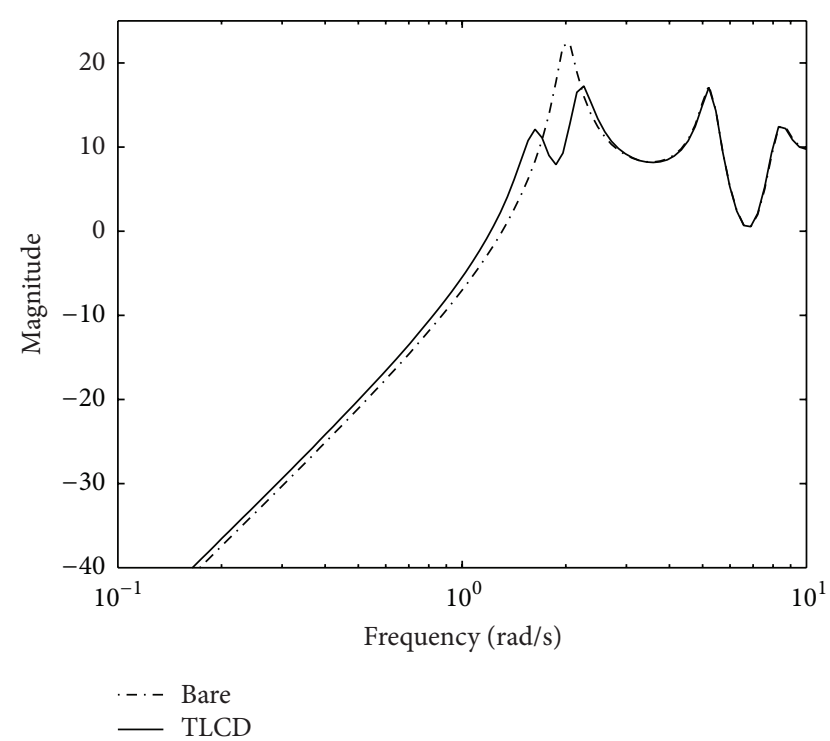

(b)

FIGURE 6: Frequency response curve comparisons: (a) frequency response for displacement; (b) frequency response for acceleration.

After the assumed TLCD is optimized, it would be divided into three same TLCDs, whose total mass ratio, frequency tuning ratio, and damping ratio are, respectively, equal to the optimized parameters. To design the TLCD, its mass ratio is pregiven to be 0.015 , which means that $m_{T}$ is $1.5 \%$ of the structural total mass. And the value ranges for its frequency tuning ratio $\tau$ and damping ratio $\zeta_{T}$ are set to be $0 \sim 2.0$ and $0 \sim 0.1$. So, the ranges for $k_{T}$ and $c_{T}$ can be derived as $0=c_{T}^{l}<$ $c_{T}<c_{T}^{u}=1.3373 \times 10^{6}$ and $0=k_{T}^{l}<k_{T}<k_{T}^{u}=4.1923 \times 10^{7}$ according to (9). The length ratio $\alpha$ in (6) is set to be 0.8 . The parametric optimizing process described in the last section is used. After the iteration of the ILMI method, the static output feedback controller $\mathbf{G}$ in (16) is obtained:

$$
\mathbf{G}=\left[\begin{array}{ll}
k_{T} & \mathcal{C}_{T}
\end{array}\right]=\left[\begin{array}{ll}
7.83 \times 10^{6} & 5.78 \times 10^{5}
\end{array}\right] .
$$

Then, the parameters $\tau$ and $\zeta_{T}$ are determined as

$$
\tau=0.86452 ; \quad \zeta_{T}=0.09988
$$

To study the TLCD performance, the frequency response comparisons between the lumped model without TLCD and that with the optimized TLCD are shown in Figure 6.

Figure 6(a) is the frequency response for the top floor displacement. The dashed line named Bare denotes the frequency response of the structural top floor displacement without TLCD, while the solid line named TLCD is for the response with TLCD. It is found that the frequency response peak is reduced by TLCD, which can reduce the vibration response in time domain indirectly. It means that the building instrumented with TLCD may satisfy the safety need more easily than that without TLCD. Figure 6(b) is the frequency response for the top floor acceleration. The dashed line and solid line have similar definition with that in Figure 6(a). It illustrates that the TLCD can reduce the vibration acceleration and make the building satisfy the comfortable requirement. The comparison in the frequency domain can reflect the inherent property of the structure with TLCD.

In practical engineering analysis, it is necessary to make some comparisons in the time domain. Some earthquake waves should be chosen to verify that the structural vibration can be reduced, subjected to any earthquake excitation. There are four earthquake waves to be chosen, which are Kobe wave, El Centro wave, North wave, and Hach wave. Their peak accelerations are scaled to $0.8337 \mathrm{~m} / \mathrm{s}^{2}, 0.3495 \mathrm{~m} / \mathrm{s}^{2}$, $0.8428 \mathrm{~m} / \mathrm{s}^{2}$, and $0.2294 \mathrm{~m} / \mathrm{s}^{2}$, respectively. Due to the space limitation, only the top floor responses are compared as shown in Figures 7 and 8.

In Figure 7, the dashed line named Bare means the structure without TLCD and the solid line named TLCD is the one with TLCD control. From this figure, all the solid line can reduce the corresponding dashed line a lot. In order to describe the reduction precisely, the reduction ratios for the top floor displacement under the different earthquake waves are calculated according to the following equation:

reduction ratio

$$
\begin{aligned}
= & \frac{(\text { Bare structural response })-(\text { TLCD structural response })}{\text { Bare structural response }} \\
& \times 100 \% .
\end{aligned}
$$

The reduction ratios are $3.13 \%, 31.31 \%, 16.13$, and $28.55 \%$ under the Kobe wave, El Centro wave, North wave, and Hach wave, respectively. It means that the TLCD can suppress the structural displacement vibration efficiently, which can decrease the component damage and make the structure safe. The acceleration comparisons are also made as shown in Figure 8. 


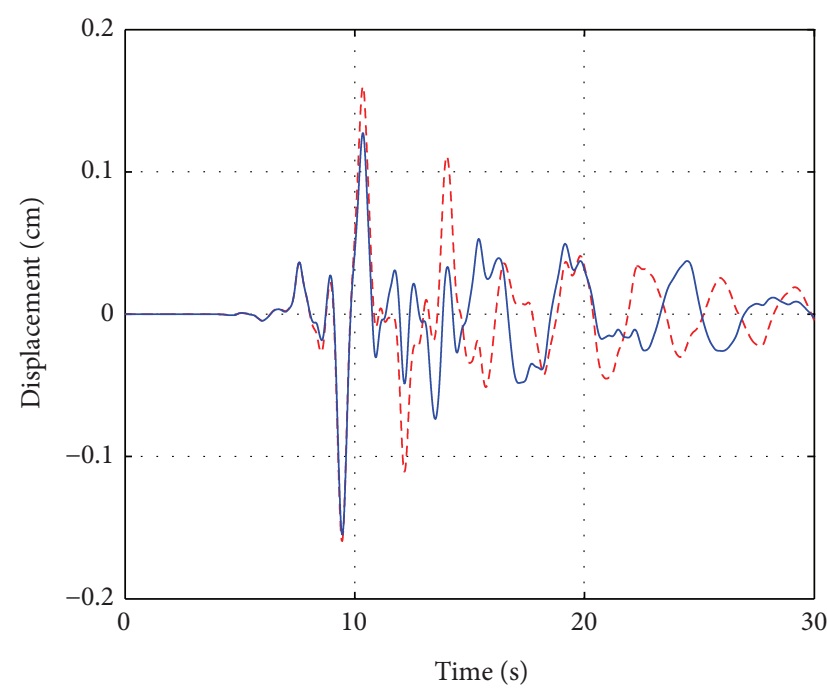

(a)

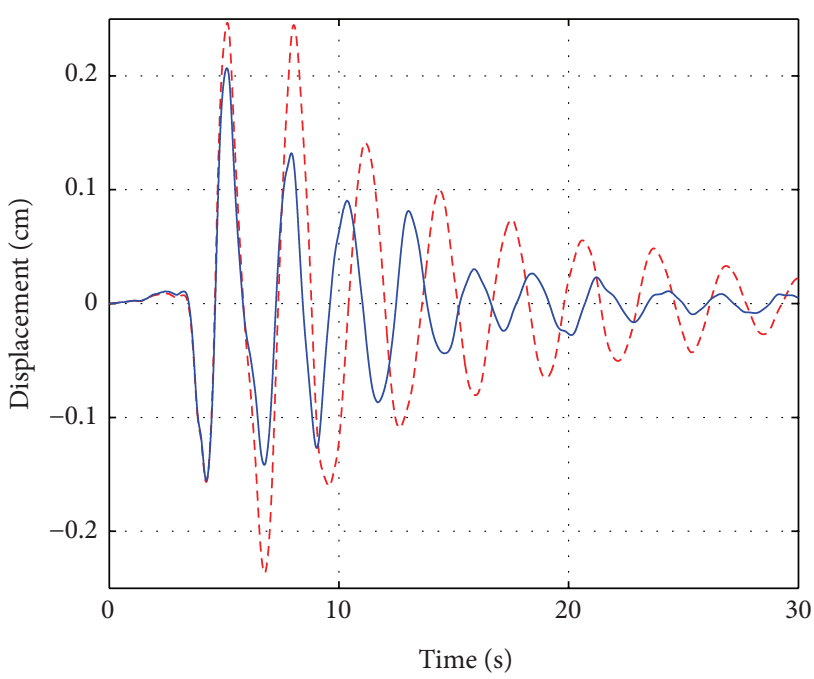

- - Bare

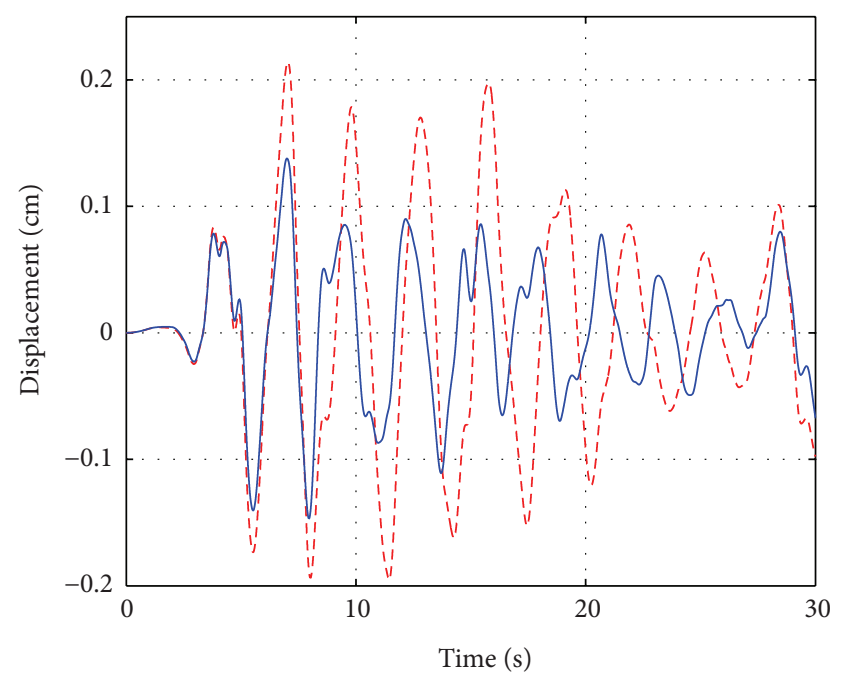

(b)

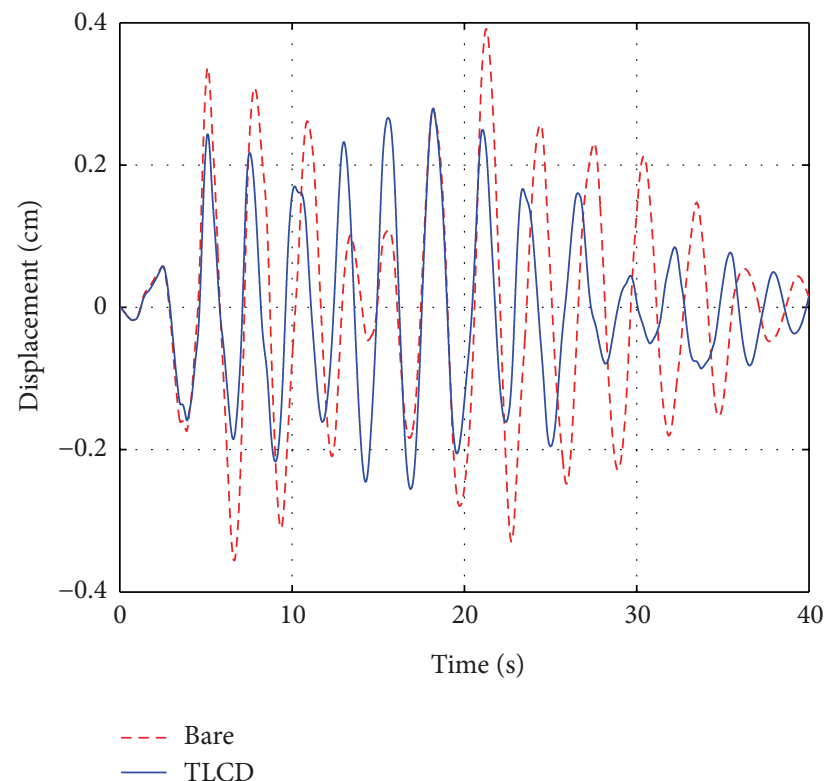

(d)

FiguRE 7: Time history comparison for the top floor displacement: (a) under Kobe wave; (b) under El Centro wave; (c) under North wave; (d) under Hach wave.

In Figure 8, the dashed line and solid line have the same definition as that in Figure 7. In the four figures, all the solid line can reduce the peak value of the corresponding dashed line. The reduction ratios for the top floor acceleration under the four earthquake waves are also calculated as (29), which are $3.54 \%, 4.05 \%, 5.09 \%$, and $8.84 \%$, respectively. It means that TLCD can make the structure satisfy the requirement for comfort.

\section{Conclusion}

This paper optimizes the parameters of TLCD for Building 1 in Dalian Xinghai Financial Business Building with the consideration of the earthquake excitation. The building is built to the finite element model and the lumped mass model, respectively. After the comparison of these two models, they are pretty close. The lumped model is adopted due to the simple calculation process for optimization.

TLCD is used to suppress the structural vibration subjected to the earthquake excitation. The TLCD parameters are considered to be optimized. Through the equation derivation, the parametric optimization problem is transferred to the feedback controller design problem. The controller is the static output feedback controller with some value range constraints. This paper designs a static $H_{\infty}$ controller. According to bounded real lemma, a bilinear matrix inequality (BMI) is constructed. To solve the BMI problem, the iterative linear 


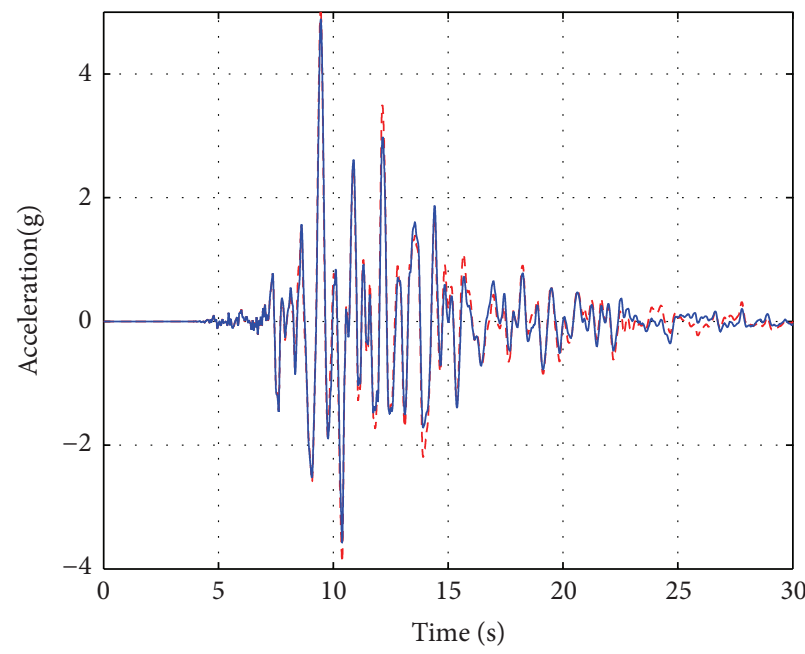

(a)

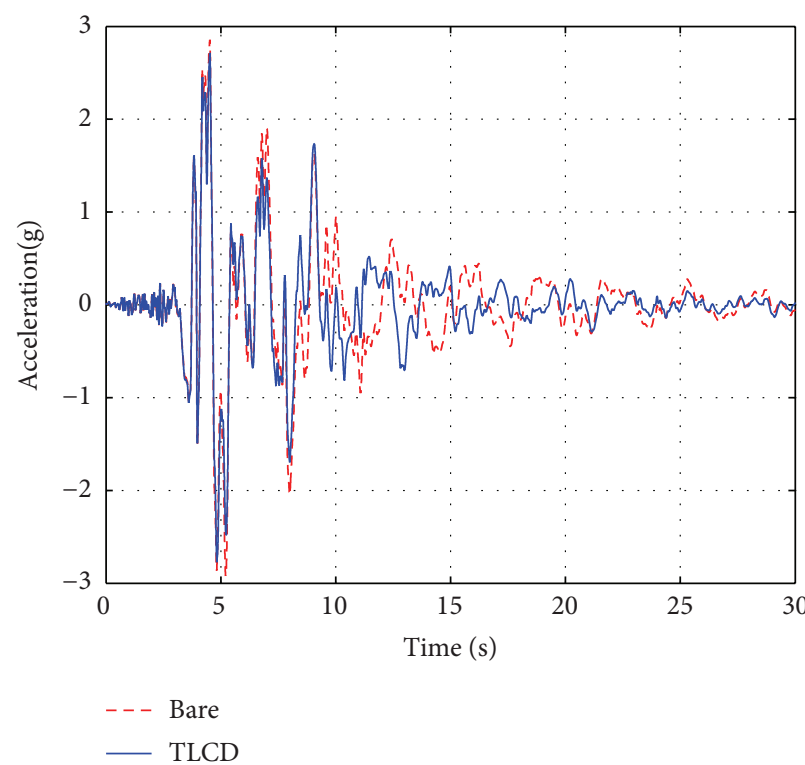

(c)

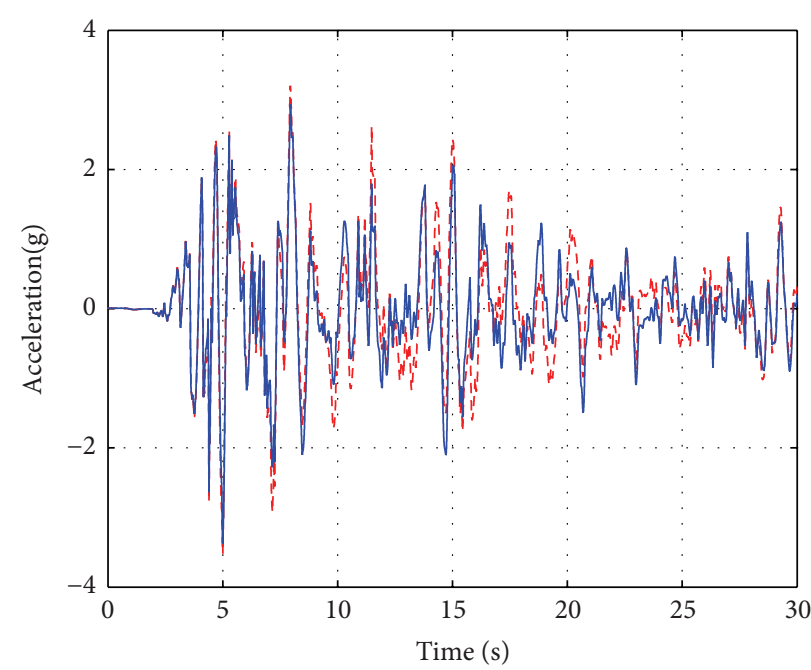

(b)

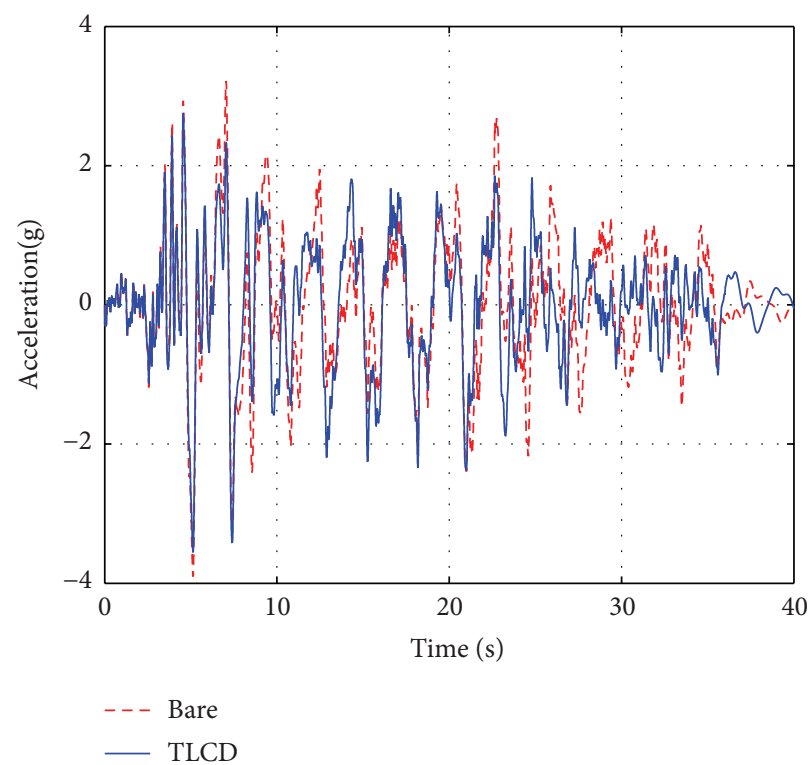

(d)

FIGURE 8: Time history comparison for the top floor acceleration: (a) under Kobe wave; (b) under El Centro wave; (c) under North wave; (d) under Hach wave.

matrix inequality (ILMI) is employed and added by the value range constraints. After the iteration, the feedback controller is calculated. Then the frequency tuning ratio and damping ratio of TLCD are determined. To illustrate the TLCD performance, the frequency responses of the top floor displacement and acceleration for the building without and with TLCD in frequency domain are compared. In time domain, the displacement and acceleration responses are compared, subjected to the four earthquake waves. It is found that TLCD can reduce both the displacements and accelerations of the building. It means that the TLCD can make the structure satisfy the need for safety and comfort.

\section{Conflict of Interests}

The authors declare that there is no conflict of interests regarding the publication of this paper.

\section{Acknowledgments}

The authors would like to thank the support of the National Natural Science Foundation of China (Grant nos. 51121005 and 51261120375). 


\section{References}

[1] G. W. Housner, L. A. Bergman, T. K. Caughey et al., "Structural control: past, present, and future," Journal of Engineering Mechanics, vol. 123, no. 9, pp. 897-971, 1997.

[2] C.-C. Lin, C.-L. Chen, and J.-F. Wang, "Vibration control of structures with initially accelerated passive tuned mass dampers under near-fault earthquake excitation," Computer-Aided Civil and Infrastructure Engineering, vol. 25, no. 1, pp. 69-75, 2010.

[3] A. S. Pall and C. Marsh, "Response of friction damped braced frames," Journal of the Structural Division, vol. 108, no. 6, pp. 1313-1323, 1982.

[4] J. T. P. Yao, "Concept of structural control," Journal of Structural Division, vol. 98, no. 7, pp. 1567-1574, 1972.

[5] F. Sakai, S. Takaeda, and T. Tamaki, "Tuned liquid column damper-new type device for suppression of building vibrations," in Proceedings of the International Conference on Highrise Buildings, Nanjing, China, 1989.

[6] C. P. Moreno and P. Thomson, "Design of an optimal tuned mass damper for a system with parametric uncertainty," Annals of Operations Research, vol. 181, pp. 783-793, 2010.

[7] U. Mackenroth, Robust Control Systems: Theory and Case Studies, Springer, Berlin, Germany, 2004.

[8] J. Rubió-Massegú, F. Palacios-Quiñonero, and J. M. Rossell, "Decentralized static output-feedback $H_{\infty}$ controller design for buildings under seismic excitation," Earthquake Engineering and Structural Dynamics, vol. 41, no. 7, pp. 1199-1205, 2012.

[9] A. I. Zečević and D. D. Šiljak, "Design of robust static output feedback for large-scale systems," IEEE Transactions on Automatic Control, vol. 49, no. 11, pp. 2040-2044, 2004.

[10] Y.-Y. Cao, Y.-X. Sun, and W.-J. Mao, "Output feedback decentralized stabilization: ILMI approach," Systems \& Control Letters, vol. 35, no. 3, pp. 183-194, 1998.

[11] Y. Wang, "Time-delayed dynamic output feedback $H_{\infty}$ controller design for civil structures: a decentralized approach through homotopic transformation," Structural Control and Health Monitoring, vol. 18, no. 2, pp. 121-139, 2011.

[12] M. Kočvara and M. Stingl, "Pennon: a code for convex nonlinear and semidefinite programming," Optimization Methods \& Software, vol. 18, no. 3, pp. 317-333, 2003. 


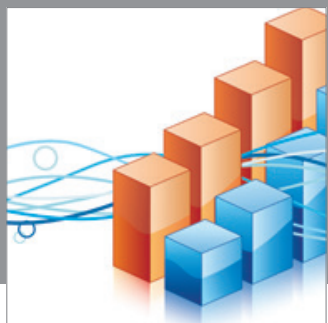

Advances in

Operations Research

mansans

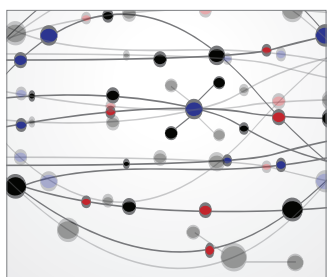

The Scientific World Journal
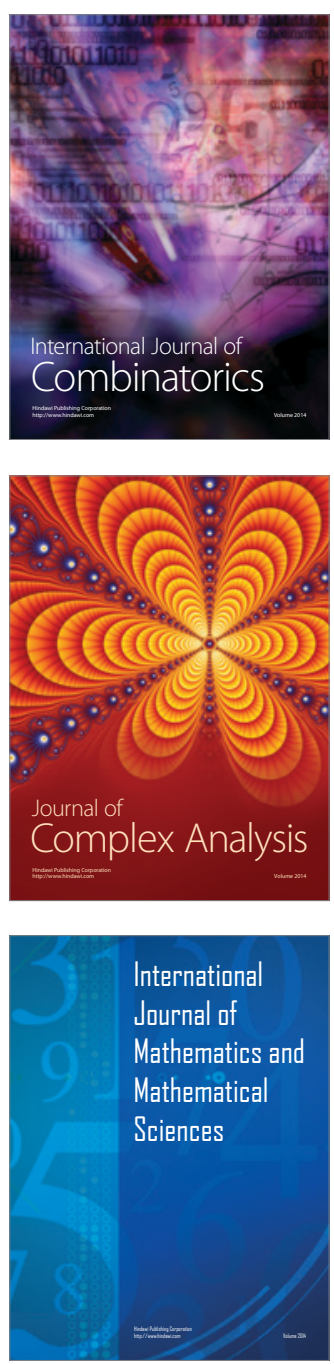
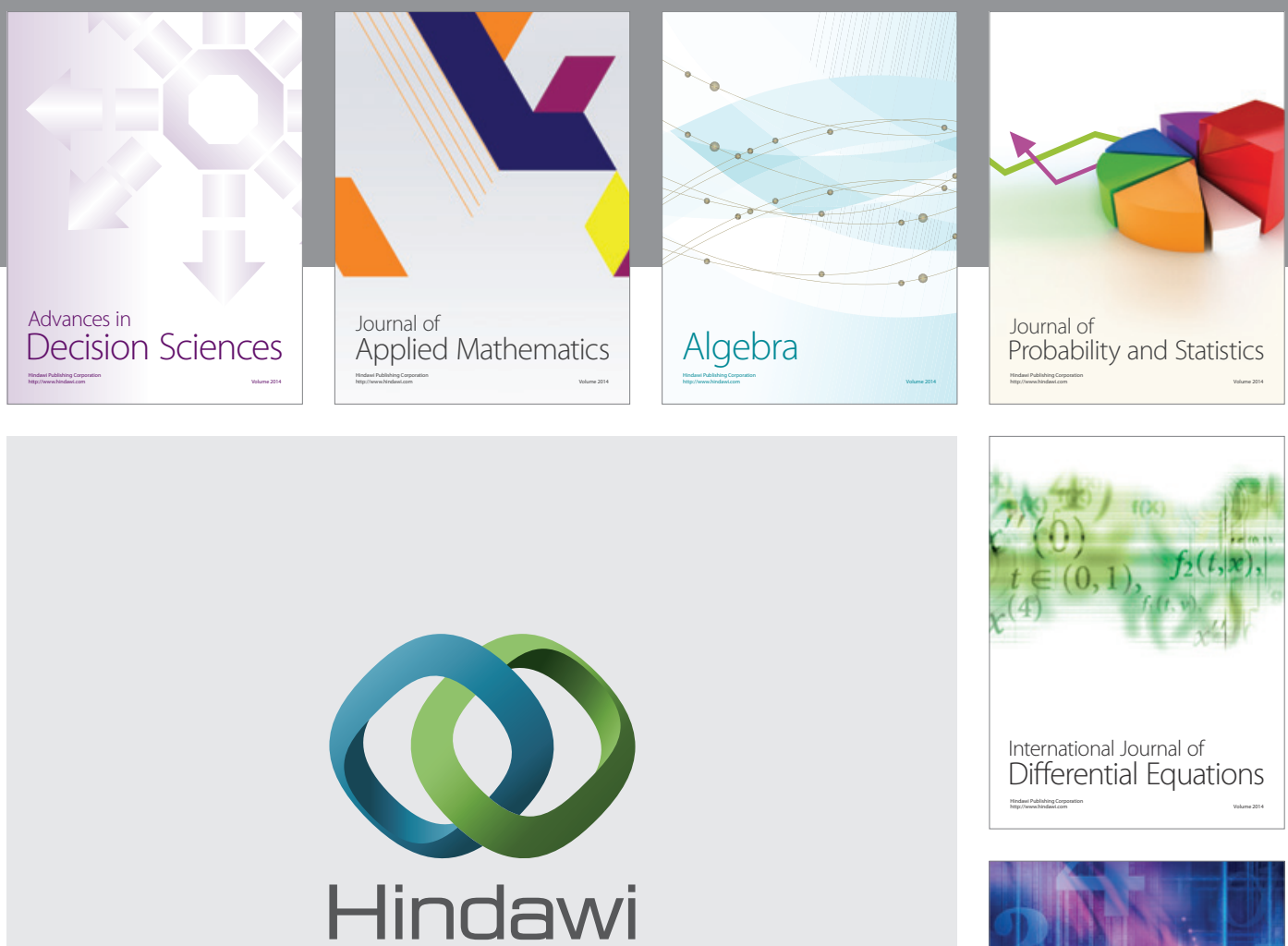

Submit your manuscripts at http://www.hindawi.com
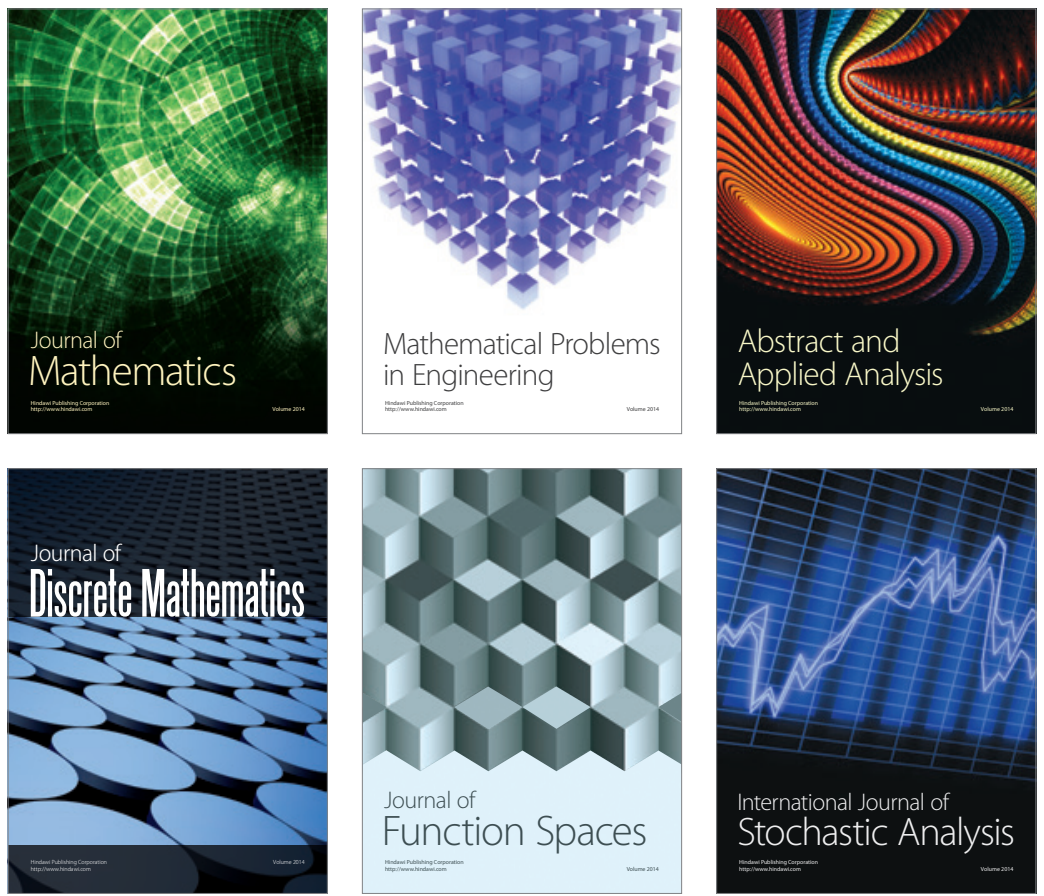

Journal of

Function Spaces

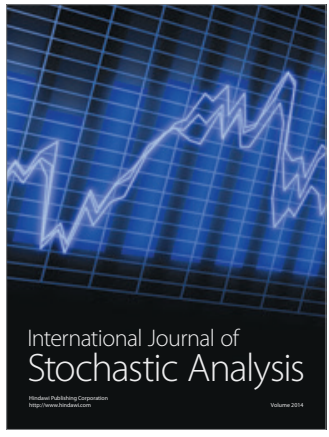

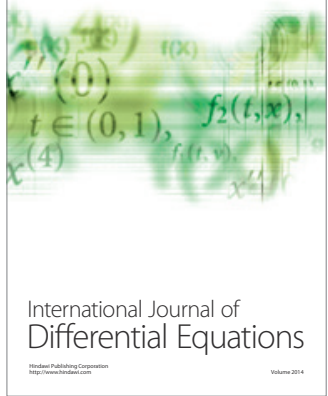
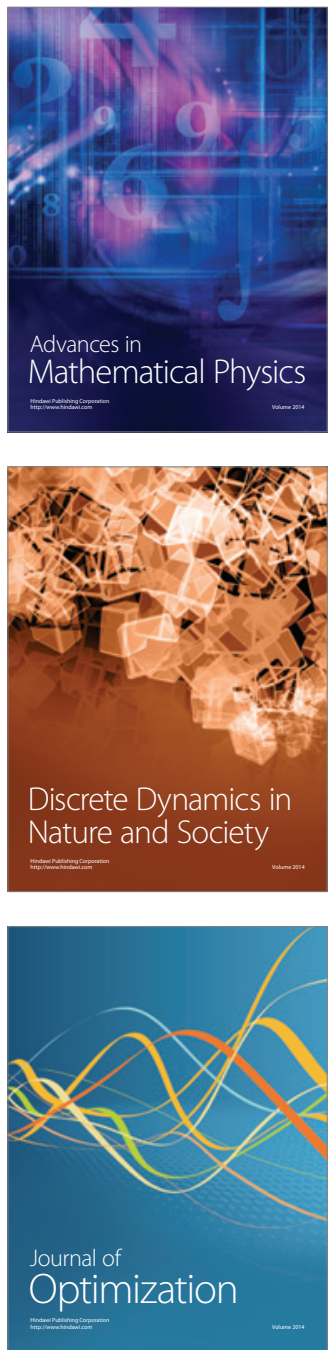